\title{
The search for quantum critical scaling in a classical system
}

\author{
Jagat Lamsal, ${ }_{2}^{1}$ John Gaddy, ${ }^{1}$ Marcus Petrovic, ${ }^{1}$ Wouter Montfrooij, ${ }^{1, a)}$ and \\ Thomas Vojta ${ }^{2}$ \\ ${ }^{1}$ Department of Physics and Missouri Research Reactor, University of Missouri, Columbia, \\ Missouri 65211, USA \\ ${ }^{2}$ Department of Physics, Missouri University of Science and Technology, Rolla, Missouri 65409, USA
}

(Presented 14 November 2008; received 16 September 2008; accepted 4 November 2008; published online 12 March 2009)

\begin{abstract}
Order-disorder phase transitions in magnetic metals that occur at zero temperature have been studied in great detail. Theorists have advanced scenarios for these quantum critical systems in which the unusual response can be seen to evolve from a competition between ordering and disordering tendencies, driven by quantum fluctuations. Unfortunately, there is a potential disconnect between the real systems that are being studied experimentally, and the idealized systems that theoretical scenarios are based upon. Here we discuss how disorder introduces a change in morphology from a three-dimensional system to a collection of magnetic clusters, and we present neutron scattering data on a classical system, $\mathrm{Li}\left[\mathrm{Mn}_{1.96} \mathrm{Li}_{0.04}\right] \mathrm{O}_{4}$, that show how magnetic clusters by themselves can lead to scaling laws that mimic those observed in quantum critical systems. (C) 2009 American Institute of Physics. [DOI: 10.1063/1.3068409]
\end{abstract}

Quantum fluctuations can be strong enough to prevent a system from ordering, even at $0 \mathrm{~K}$. In metals that possess atomic magnetic moments, one can tweak the strength of the magnetic interactions compared to the disordering quantum fluctuations in such a way that the system orders exactly at 0 $\mathrm{K}$. Such a system is said to be at the quantum critical point (QCP). The interest in such systems is easy to understand. One can expect a new type of ordering behavior because the spatial and temporal dimensions are no longer independent at $0 \mathrm{~K} .{ }^{1}$ Also, one can expect new physics to emerge. After all, when a system is on the verge of ordering at $0 \mathrm{~K}$, the degrees of freedom that prevent the system from ordering also provide a channel for the system to adopt a new, lower energy ground state. An example is the observed superconducting state $^{1}$ that forms close to the QCP with quasiparticles consisting of some admixture of magnetic moments and conduction electrons.

The question that has attracted most attention is "what exactly happens at the QCP?" On the one hand, ${ }^{2}$ it could be that all moments will become fully shielded at some finite temperature, with the residual interaction between the resulting heavy quasiparticles driving the system toward ordering [Fig. 1(a)]. On the other hand, ${ }^{2}$ the QCP could be the point in the phase diagram where vestiges of moments can survive all the way down to $0 \mathrm{~K}$ upon cooling without being completely screened though the Kondo effect, resulting in long-range order [Fig. 1(b)]. Unfortunately, the experimental situation is much murkier than a simple choice between these two possible answers.

To drive a system to a QCP, one tweaks the interaction between moments by changing the degree of overlap between the local atomic orbitals and the extended conduction electron bands. Ideally, one simply applies hydrostatic pressure to a system without any intrinsic disorder, and all mag-

${ }^{\text {a)} E l e c t r o n i c ~ m a i l: ~ m o n t f r o o i j w ~ @ ~ m i s s o u r i . e d u . ~}$ netic moments will undergo the same temperature evolution. This is the situation that most theoretical efforts have focused on [Ref. 1]. In some experiments however, one cannot attain the high pressures required and one results to applying chemical pressure. Here, some elements are substituted with different sized ones so as to achieve lattice expansion/ contraction at the cost of introducing some disorder. However, it was generally believed that controlled amounts of disorder should not affect the outcome too much. Unfortunately, the study of $\mathrm{Ce}\left(\mathrm{Ru}_{1-x} \mathrm{Fe}_{x}\right)_{2} \mathrm{Ge}_{2}$ revealed ${ }^{3}$ that this is not the case. From a chemical substitution point of view, this system is as clean as any system that one can study. $\mathrm{CeFe}_{2} \mathrm{Ge}_{2}$ is a heavy fermion system ${ }^{4}$ which upon increased doping $x$ of $\mathrm{Ru}$ on the $\mathrm{Fe}$ sites orders magnetically at $0 \mathrm{~K}$ once 1:4 Fe ions have been substituted. ${ }^{3}$ Since this doping is being done on sites that are not nearest neighbors to the moment carrying Ce sites, and since this substitution is isovalent, this system should have yielded unambiguous results.

Instead, neutron scattering experiments showed ${ }^{3}$ that this type of doping necessarily must lead to the formation of a magnetic percolation network. The reason for this is rather straightforward. When chemical doping takes place, locally

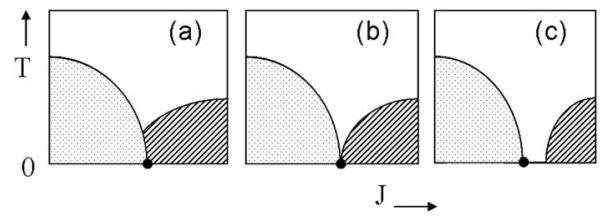

FIG. 1. The possibilities for the phase diagram near the QCP (black dot) as a function of the coupling strength $J$ between magnetic moments and conduction electrons. In (a) the moments are fully shielded for large $J$ by the conduction electrons (striped area) at finite $T$ and ordering (light gray area) near the QCP is driven by a spin-density wave. In (b) the QCP is where moments first survive down to $0 \mathrm{~K}$ (Ref. 2). In (c) a spread in shielding temperatures results in a region in between the areas where the moments order and where they are fully shielded. Here, some moments survive down to $0 \mathrm{~K}$ but they are located in clusters that are not interconnected. 
the interatomic distances are changed by a very small amount, typically on the order of $0.05 \AA^{-1}$. While this is a small number, it is enough to locally change the overlap between neighboring orbitals to such an extent that it will introduce a distribution of temperatures at which the moments become fully shielded. Upon cooling, some magnetic moments will be shielded before others, resulting in a percolation network that follows the random chemical doping sites. This is unavoidable since in a system that is on the verge of ordering any change, no matter how small, will make a difference. Such a system cannot be described as a collection of magnetic moments that all undergo shielding at the same temperature; rather, one has to take into account the breaking up into smaller clusters upon cooling, especially if this takes the system close to the percolation threshold.

The experimental signature of such a network is provided by the extent of the short range magnetic ordering along the various crystallographic directions. A fractal structure would show up as a frozen arrangement of magnetic moments, randomly distributed along various clusters. The magnetic correlation lengths would span equal numbers of moments in all directions, independent of the actual distances between the magnetic ions along these directions. The existence of such a percolation network was verified ${ }^{5}$ in tetragonal $\mathrm{Ce}\left(\mathrm{Ru}_{1-x} \mathrm{Fe}_{x}\right)_{2} \mathrm{Ge}_{2}$, where it was observed that moment correlations do indeed span equal numbers of $\mathrm{Ce}$ ions in all directions, despite the large $c / a$ ratio of 2.5.

Therefore, the phase diagram of such quantum critical systems is likely more complicated than normally presumed (Fig. 1). There is a region in between the ordered phase and the phase where all moments are fully shielded, in which some moments survive (and order) in isolated clusters that form at random upon cooling. Thus, the system slowly undergoes a transition from a fully connected threedimensional network of magnetic moments, to a fractal structure characterized by clusters. In this paper we investigate whether it is possible that the emergence of unusual dynamical scaling behavior in quantum critical systems $[E / T$ scaling] is not independent of the emergence of the percolation network. For this purpose we measure the response in a classical system that is known to harbor magnetic clusters.

We have been studying the approach to magnetic ordering in the cubic spinel structure $\mathrm{LiMn}_{2} \mathrm{O}_{4}$. This compound, which is of interest in its own right because of its use in Li-based batteries, has an equal amount of $\mathrm{Mn}^{3+}$ and $\mathrm{Mn}^{4+}$ ions arranged in corner sharing tetrahedra. Below the metalinsulator transition at room temperature, the $\mathrm{Mn}^{4+}$ ions form eightfold ring clusters. ${ }^{6}$ The main interaction between all $\mathrm{Mn}$ ions is antiferromagnetic, resulting in a frustrated structure. Nonetheless, when this system is cooled down to below 66 $\mathrm{K}$, long-range magnetic order is seen to emerge, ${ }^{7}$ the details of which have not been resolved yet.

It was discovered ${ }^{8}$ that by substituting around 1:50 Mn ions for $\mathrm{Li}$ ions that an unwanted (from a battery applications' point of view) room temperature structural phase transition could be suppressed. Neutron scattering experiments showed $^{5,8,9}$ that while long-range order does not emerge down to $4 \mathrm{~K}$ in the doped sample, the $\mathrm{Mn}^{4+}$ ions in the eightfold rings line up antiferromagnetically (AF) below 66

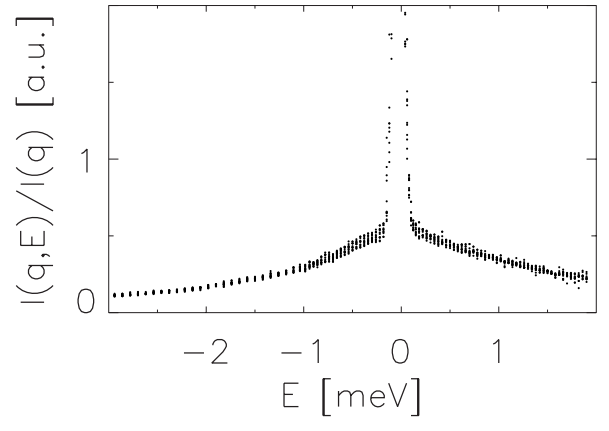

FIG. 2. The energy dependence of the scattered intensity $I(q, E)$ for 12 $q$-values $0.8<q<1.9 \AA^{-1}$ at $T=50 \mathrm{~K}$. The energy resolution is given by the sharp central line due to incoherent nuclear scattering. After normalizing to $I(q)=\int_{0.6}^{1} I(q, E) d E$, all data points collapse onto a single curve, showing that the time scale of the dynamics is independent of $q$. The figure contains 1600 independent data points.

$\mathrm{K}$ (the undoped sample shows $\mathrm{AF}$ order $^{7}$ at $\sim 66 \mathrm{~K}$ ). Since the structure factor associated with the $\mathrm{Mn}^{4+}$ scattering does not change ${ }^{5}$ between 4 and $70 \mathrm{~K}$, we can assume that AF order is present within the rings in this temperature range, and that the rings behave as so-called superspins. The dynamics of these superspins appears ${ }^{5,9}$ to freeze out below $\sim 25 \mathrm{~K}$. The $\mathrm{Mn}^{3+}$ ions do not freeze out down to $4 \mathrm{~K}$. Thus, $\mathrm{Li}\left[\mathrm{Mn}_{1.96} \mathrm{Li}_{0.04}\right] \mathrm{O}_{4}$ is a purely classical system (from a phase transition point of view) that is disordered because of geometric frustration and $\mathrm{Li} / \mathrm{Mn}$ disorder with clusters of $\mathrm{AF}$ aligned $\mathrm{Mn}^{4+}$ ions (superspins) present below $66 \mathrm{~K}$.

Insulating $\mathrm{Li}\left[\mathrm{Mn}_{1.96} \mathrm{Li}_{0.04}\right] \mathrm{O}_{4}$ should not exhibit any type of quantum critical scaling, however, next we argue that the response shows the hallmarks of $E / T$ scaling that were thought to be associated exclusively with quantum criticality. We argue, without using any particular line shape analysis, that scattering originating from the superspins effectively mimics quantum scaling behavior.

The neutron scattering experiments were carried out using the IN6 time-of-flight chopper spectrometer at the Institute Laue-Langevin (ILL), and using the TRIAX triple-axis spectrometer at the Missouri Research Reactor MURR. The ILL experiments and data reduction are described in detail elsewhere. ${ }^{5}$ For the MURR experiments $\mathrm{Li}\left[\mathrm{Mn}_{1.96} \mathrm{Li}_{0.04}\right] \mathrm{O}_{4}$ powder was placed in a slab sample cell which was housed inside a closed cycle refrigerator. The spectrometer was operated at fixed final energy $E_{f}$ of $13.7 \mathrm{meV}$, with sapphire and PG/Si filters in the incoming beam, and PG filters in the scattered beam. The nuclear scattering and sample holder scattering were removed from the spectra by comparison to the data at $150 \mathrm{~K}$.

In Fig. 2 we show that the dynamics are independent of probing wavelength $\lambda$ in the range $0.8 \AA^{-1}<q=2 \pi / \lambda$ $<2 \AA^{-1}$. We have taken the ILL data, ${ }^{5}$ and divided the energy scans $I(q, E)$ at constant $q$ by the total scattering at that particular $q$-value in the range $0.6<E<1 \mathrm{meV}$. The $q$-dependence of the scattering in this range essentially follows a magnetic form factor based on eightfold rings ${ }^{9}$ and allows for a $q$-normalization that is independent of resolution effects. The resulting curves are plotted in Fig. 2, without any further data correction. The fact that all curves coincide implies that the dynamics are $q$-independent on these length 

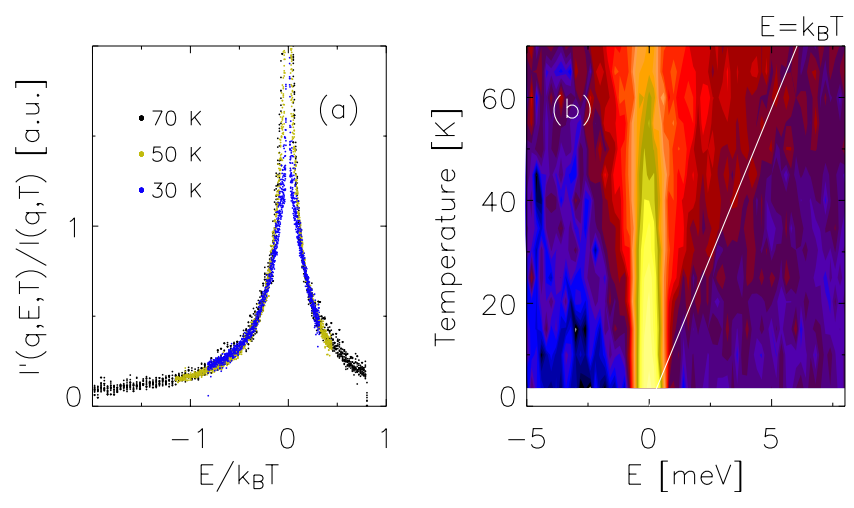

FIG. 3. (Color online) (a) Same as Fig. 2, but now for $T=30 \mathrm{~K}, 50$ and 70 $\mathrm{K}$, plotted vs $E / k_{B} T$ with the Bose population factor taken out. All curves collapse onto each other, giving the appearance of dynamical scaling. The differences for $\left|E / k_{B} T\right|<0.1$ are due to resolution effects. (b) The scattering profile at $q=1.4 \AA^{-1}$ follow straight lines $E \sim T$ showing that the scattering develops as if $E / T$ is the only relevant variable. The white line is $E=k_{B} T$.

scales, as is to be expected when probing superspins on length scales smaller than the distance between clusters $d_{c c}$ $\sim 8 \AA$ (or $q=2 \pi / d_{c c}>0.8 \AA^{-1}$ ). Combined with the fact that the static magnetic structure factor is well described by that of eightfold $\mathrm{Mn}^{4+}$ ring clusters, we conclude that the dynamics in $\mathrm{Li}\left[\mathrm{Mn}_{1.96} \mathrm{Li}_{0.04}\right] \mathrm{O}_{4}$ are those of a system of superspins.

When we plot our results as a function of $E / T$ we find that our data mimic the $E / T$-scaling behavior normally associated with quantum criticality. In Fig. 3(a) we plot the scattering for $T=30,50$, and $70 \mathrm{~K}$. To be consistent with the $E / T$-like scaling that we will show, we used $0.6(T / 50 \mathrm{~K})$ $<E<(T / 50 \mathrm{~K}) \mathrm{meV}$ as the energy range for the $q$-normalization. All $\sim 5000$ independent data points collapse onto a single curve, independent of any line shape analysis or model. In Fig. 3(b) we show the TRIAX data at $q$ $=1.4 \AA^{-1}$ for $5<T<70 \mathrm{~K}$. The energy and temperature dependence of the scattering follows curves that are functions of $E / T$ only (straight lines in the figure) and therefore, the scattering associated with the superspins could easily be interpreted as exhibiting $E / T$ scaling such as observed in $\mathrm{CeCu}_{5.9} \mathrm{Au}_{0.1},{ }^{2} \mathrm{UCu}_{5-x} \mathrm{Pd}_{x}{ }^{10}$ and $\mathrm{Ce}\left(\mathrm{Ru}_{1-x} \mathrm{Fe}_{x}\right)_{2} \mathrm{Ge}_{2} .{ }^{3}$ Thus, the dynamics of this classical system of clusters are annoyingly similar to what one observes in doped quantum critical systems. This similarity is particularly strong in the case of heavily doped $\mathrm{UCu}_{5-x} \mathrm{Pd}_{x}(x=1,1.5)^{10}$ where the static structure factor closely follows that of our system, ${ }^{5}$ and where the dynamics do not show any significant $q$-dependence when plotted as $S(q, E, T) / S(q, T)$ as we did in Fig. 3. This could very well imply that magnetic clusters have also formed in $\mathrm{UCu}_{5-x} \mathrm{Pd}_{x}$, obfuscating the distinction between classical physics and a quantum critical response.

In conclusion, we have shown that the dynamics in $\mathrm{Li}\left[\mathrm{Mn}_{1.96} \mathrm{Li}_{0.04}\right] \mathrm{O}_{4}$, a system far removed from any quantum critical point and characterized by the thermally activated response of the superspins of the $\mathrm{Mn}^{4+}$ clusters, effectively reproduces $E / T$-scaling behavior. Given the high likelihood ${ }^{3}$ that quantum critical systems prepared by means of substantial chemical doping ${ }^{5,10}$ will fragment into a collection of magnetic clusters upon cooling, it appears that in describing the physics at a QCP one should take into account the inherent change in sample morphology when a system is cooled to $0 \mathrm{~K}$.

This material was based upon work supported by the Department of Energy under Award No. DE-FG0207ER46381 and by the University of Missouri Research Board (Grant No. RB-07-52).

${ }^{1}$ G. R. Stewart, Rev. Mod. Phys. 73, 797 (2001).

${ }^{2}$ A. Schröder, G. Aeppli, R. Coldea, M. Adams, O. Stockert, H. v. Löhneysen, E. Bucher, R. Ramazashvili, and P. Coleman, Nature (London) 407, 351 (2000).

${ }^{3}$ W. Montfrooij, M. C. Aronson, B. D. Rainford, J. A. Mydosh, A. P. Murani, P. Haen, and T. Fukuhara, Phys. Rev. Lett. 91, 087202 (2003); W. Montfrooij, J. Lamsal, M. Aronson, M. Bennett, A. de Visser, H. Y. Kai, N. T. Huy, M. Yethiraj, M. Lumsden, and Y. Qiu, Phys. Rev. B 76, 052404 (2007).

${ }^{4}$ M. B. Fontes, M. A. Continentino, S. L. Bud'ko, M. El-Massalami, L. C. Sampaio, A. P. Guimarães, E. Baggio-Saitovitch, M. F. Hundley, and A. Lacerda, Phys. Rev. B 53, 11678 (1996).

${ }^{5}$ H. G. Schimmel, W. Montfrooij, G. J. Kearley, V. W. Verhoeven, and I. M. de Schepper, Phys. Rev. B 63, 214409 (2001).

${ }^{6}$ J. Rodriguez-Carvajal, G. Rousse, C. Masquelier, and M. Hervieu, Phys. Rev. Lett. 81, 4660 (1998)

${ }^{7}$ J. E. Greedan, C. R. Wiebe, A. S. Wills, and J. R. Stewart, Phys. Rev. B 65, 184424 (2002).

${ }^{8}$ V. W. J. Verhoeven, F. M. Mulder, and I. M. de Schepper, Physica B 276-278, 950 (2000).

${ }^{9}$ J. Gaddy, J. Lamsal, M. Petrovic, W. Montfrooij, A. Schmets, and T. Vojta, "Magnetic ordering in the spinel compound $\mathrm{Li}\left[\mathrm{Mn}_{2-x} \mathrm{Li}_{x}\right] \mathrm{O}_{4}(x$ $=0,0.04)$," J. Appl. Phys. (these proceedings).

${ }^{10}$ M. C. Aronson, R. Osborn, R. Chau, M. B. Maple, B. D. Rainford, and A. P. Murani, Phys. Rev. Lett. 87, 197205 (2001). 